\title{
Bipolar II disorder as the initial presentation of CADASIL: an underdiagnosed manifestation
}

\author{
This article was published in the following Dove Press journal: \\ Neuropsychiatric Disease and Treatment \\ 14 August 2017 \\ Number of times this article has been viewed
}

\section{Jianjun Wang' \\ Jinfang $\mathrm{Li}^{2}$ \\ Fanxin Kong ${ }^{2}$ \\ Hanqing $L v^{3}$ \\ Zhouke Guo²}

'Department of Neurology and Psychology, the Fourth Clinical College, Guangzhou University of Chinese Medicine, Shenzhen, Guangdong, People's Republic of China; ${ }^{2}$ Department of Neurology and Psychology, Shenzhen Hospital of Chinese Medicine, Shenzhen, Guangdong, People's Republic of China; ${ }^{3}$ Medical Imaging Department, Shenzhen Hospital of Chinese Medicine, Shenzhen, Guangdong, People's Republic of China
Correspondence: Zhouke Guo Department of Neurology and Psychology, Shenzhen Hospital of Chinese Medicine, I Fuhua Road, Shenzhen 5I8033, Guangdong, People's Republic of China Tel +86755 88359666 Fax +8675588356033 Email szzyygzk@126.com

\begin{abstract}
Mood disturbances have been documented in cerebral autosomal dominant arteriopathy with subcortical infarcts and leukoencephalopathy (CADASIL). The highly varied morbidity indicates that the affective symptoms in CADASIL have not been cataloged systematically, leading to ineffective treatment, affecting the patients' quality of life, and possibly resulting in suicide. We present a case of CADASIL with bipolar II disorder as the first manifestation. A middle-aged female reported recurrent depressive episodes and appeared treatment resistant to adequate dosages and durations of antidepressants. Following a structured psychiatric interview and neuropsychological assessment, a past episode of hypomania was identified. Added treatment with sodium valproate alleviated most symptoms. Considering late-onset bipolar disorder with unexplained decline in cognition, a medical history of migraine, and a suspected family history of stroke, further cranial magnetic resonance imaging scan was performed and revealed severe leukoencephalopathy, prompting further investigation. The diagnosis was revised to CADASIL after Arg587Cys NOTCH3 mutation was confirmed. This case highlights the evolving process of affective disorder diagnosis and underlying organic etiologies. Based on the overlap of white matter hyperintensities, NOTCH3 mutation, and valproate therapy in bipolar disorder and CADASIL, bipolar II depression may be a poorly recognized manifestation of CADASIL. Well-designed clinical trials are warranted to verify the current findings.
\end{abstract}

Keywords: leukoencephalopathy, bipolar II disorder, hypomania, NOTCH3, white matter hyperintensities

\section{Introduction}

Cerebral autosomal dominant arteriopathy with subcortical infarcts and leukoencephalopathy (CADASIL), caused by mutations in the NOTCH3 gene on chromosome (19p13.2-p13.1), is a rare monogenic disorder known to cause recurrent transient ischemic attacks, strokes, and migraines (with or without aura). ${ }^{1,2}$ Apart from the neurologic manifestations, neuropsychiatric manifestations mainly comprising episodes of mood disturbances are another recognized feature of CADASIL. ${ }^{1,3}$ Morbidity due to affective symptoms during the course of CADASIL varies from $10 \%$ to $74 \%{ }^{4-6}$ Assessments of the psychiatric disturbances in this disease may be limited, risking an underestimation of these affective states. ${ }^{5,6}$ Additionally, if mood disturbances are the initial presenting symptoms or the chief complaint, it may lead to diagnosing the patient with the mood disorder only, and recognition of the underlying diagnosis of CADASIL may be delayed or missed in the psychiatric work-up, underestimating the frequency of affective symptoms in CADASIL. ${ }^{7,8}$

Bipolar II disorder is characterized by at least one episode of hypomania and at least one episode of major depression. ${ }^{9}$ Making a precise diagnosis is difficult because the symptoms of hypomania are often mistakenly treated as high-functioning behavior 
or attributed to personality. ${ }^{10}$ Lack of a specific treatment for hypomanic episodes in patients with CADASIL could result if they present with only mild symptoms. A diagnosis of bipolar disorder could be missed in these patients. ${ }^{5}$ A timely diagnosis is of great importance because bipolar II disorder is associated with a greater risk of suicidal thoughts and behaviors than unipolar depression. ${ }^{11,12}$

To our knowledge, relatives of patients with bipolar disorder are more likely to develop bipolar symptoms than those of unipolar depression patients. ${ }^{13}$ Increasingly, studies of bipolar disorder have utilized neuroimaging. ${ }^{14}$ Similar white matter hyperintensities and Notch genetics have been described in bipolar disorder and CADASIL, suggesting that the two conditions may be related. In this paper, a Chinese female with CADASIL initially presented with bipolar II disorder symptoms; we will characterize the comprehensive clinical and psychiatric aspects of the disease. The patient received a full description of the study and provided written informed consent permitting publication of case details and accompanying images.

\section{Case presentation}

Ms C, a 46-year-old female, presented to the Department of Neurology and Psychology complaining of loss of interest and "whole body" discomfort, occurring over the past 4 years. She first suffered from a depressed mood in her 40s and it recurred almost every year. She became sensitive and felt uncomfortable, as if insects were crawling around under her skin. She had multiple somatic pain symptoms and became irritable. Her condition got much worse when she felt total despair with the onset of sudden suicidal thoughts, but had no suicidal plans or attempts. She was prescribed courses of different antidepressants, including fluoxetine $(40 \mathrm{mg} / \mathrm{d})$, paroxetine $(30 \mathrm{mg} / \mathrm{d})$, sertraline $(100 \mathrm{mg} / \mathrm{d})$, and escitalopram $(20 \mathrm{mg} / \mathrm{d})$, respectively, starting 3 years prior to admission. None of these drug regimens resolved her symptoms completely, despite taking them consistently at adequate dosages and for appropriate durations.

On admission to the psychology clinic at the Shenzhen Hospital of Chinese Medicine, we performed a detailed psychiatric interview. Ten years prior to admission, the patient had experienced a brief period of unusually elevated mood without inducement, during which she was very hyperactive and energetic with rapid and pressured speech. These symptoms resulted in behavioral changes, but did not cause major impairments to daily life or work. Following the initiation of depressive symptoms, she developed mild migraines without aura that occurred periodically, especially during episodes of depressed mood and fatigue. Ms C's other medical history was unremarkable, apart from a cesarean section 23 years prior to admission. Her father, who had taken antidepressants in adulthood, died suddenly at age 55 from a suspected stroke.

The patient's physical and neurologic evaluation values were within normal limits. Her mental condition showed no evidence of delusions, hallucinations, or mania, but she had evidence of depressed mood, feelings of worthlessness, lack of interest, fatigue, increased appetite, sleep disturbances, and anxiety. She demonstrated normal insight into her mood changes and behavior. Neuropsychological testing revealed that cognitive tests were inconsistent between Chinese Mini-Mental State Examination 28/30 and Montreal Cognitive Assessment-Chinese 24/30, with a slight impairment in executive function detected. The Hamilton Depression Scale (34/68) and the Hamilton Anxiety Scale (25/56) indicated relatively severe depression and moderate anxiety. The Hypomania Check List-32 score (15/32) demonstrated occurrence of an episode of hypomania.

She was diagnosed with bipolar II disorder according to the criteria of the Diagnostic and Statistical Manual of Mental Disorders, Fifth Edition. After evidence of low compliance with lithium treatment was obtained, she was prescribed sodium valproate $(1,000 \mathrm{mg} / \mathrm{d})$, which resolved the majority of her problems including the migraines. The patient remains stable on monotherapy with sodium valproate (750 mg/d) in follow-up.

After late-onset bipolar disorder with atypical features was identified, a cranial magnetic resonance imaging (MRI) scan was performed, which revealed severe leukoencephalopathy. High signal intensity of the periventricular white matter, bilateral centrum semiovale, and right external capsule was found by fluid-attenuated inversion-recovery images (Figure 1A-E); anterior temporal poles were not involved (Figure 1F). The clinical features and MRI findings were highly suggestive of CADASIL, so genetic testing for NOTCH3 mutations was performed. As expected, a heterogeneous mutation was detected in this patient that produced an arginine to cysteine substitution in exon 11 of chromosome 19p13.2-13.1 (p.Arg587Cys). Results of routine laboratory tests including routine bloods, human immunodeficiency virus antibodies, and thyroid function were negative. The diagnosis was revised to CADASIL and bipolar II disorder.

\section{Discussion}

This case report describes an individual with bipolar depressive illness rather than the more common neurologic presentations of CADASIL. This underdiagnosed manifestation 

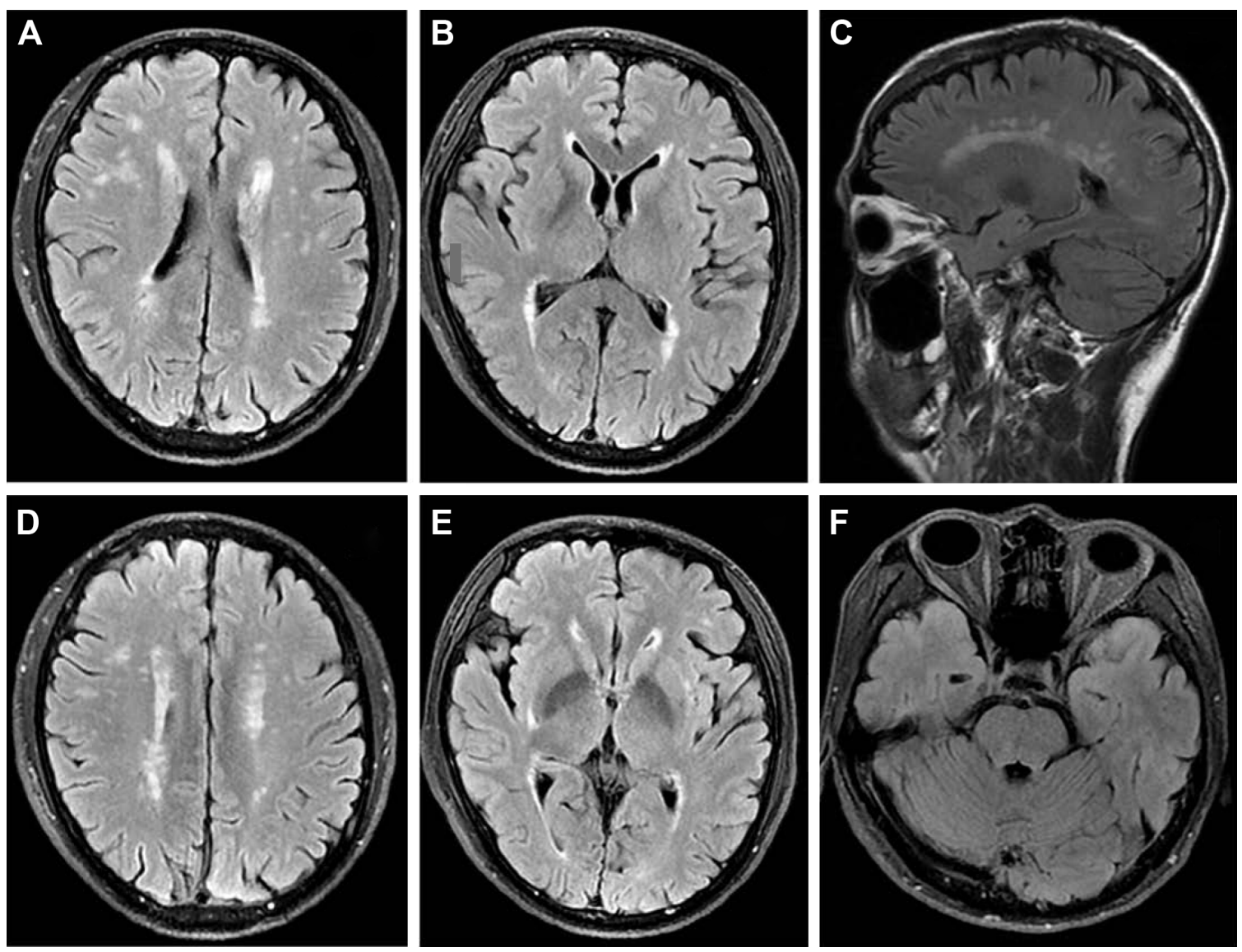

Figure I Cerebral magnetic resonance imaging with fluid-attenuated inversion-recovery (FLAIR) showing high-signal intensity lesions in the periventricular white matter $(\mathbf{A}-\mathbf{C})$, bilateral centrum semiovale (D), and right external capsule $(\mathbf{E})$, which are not prominent in the anterior temporal poles $(\mathbf{F})$.

provides new insights into the many clinical variations of CADASIL.

This study highlights the importance of a detailed psychiatric assessment and cranial MRI for an early diagnosis of CADASIL, especially when affective symptoms develop before a stroke. The diagnosis of bipolar disorder is clear, but atypical features such as late onset, minor cognitive dysfunction, and resistance to antidepressants may implicate an unusual etiology. The combination of accessible MRI scanning and deterministic genetic testing led to the final diagnosis of CADASIL. There is strong evidence that our strategy was effective and appropriate. In comparison to this outcome in a 46 year old, the confirmation of an identical Arg587Cys mutation in another recent study was delayed to the ages of 60 and 62 , respectively. ${ }^{15}$

Bipolar II disorder, with hallmark symptoms of hypomania during only $1.3 \%$ of the entire course, ${ }^{16}$ is misdiagnosed as unipolar depression in $40 \%-50 \%$ of cases. ${ }^{9}$ Thus, untoward consequences, including inappropriate medication regimens, may lead to increased mania and suicide risk, as well as decreased quality of life. ${ }^{17}$ Moreover, the psychiatric disturbances reported in CADASIL vary greatly, ${ }^{6}$ with depression being the best known, causing a risk of underestimating the presence of mania or hypomania. One possible reason may be that nonpsychiatric specialists may attach greater importance to neurologic symptoms and then provide limited descriptions and evaluations of the psychiatric symptoms. ${ }^{5}$ Valenti et al were the first to address mood disorders in CADASIL with systematic assessments and reported a lifetime prevalence of bipolar disorders of $26 \%$, which argues for recognition of depressive, hypomanic, and manic episodes as usual manifestations in patients with CADASIL.

The continuity and overlap in white matter hyperintensities described in bipolar disorder and CADASIL indicate the striking connection of mood disturbances to CADASIL. Recently, white matter hyperintensities have been utilized as an endophenotype in bipolar disorder. ${ }^{18,19}$ Brain morphometric biomarkers using white matter density data distinguish bipolar from unipolar depression and healthy controls with up to $79.3 \%$ and $70.3 \%$ accuracy, respectively. ${ }^{20,21}$ Most similar white matter lesions, involved in both CADASIL and bipolar disorder, ${ }^{22,23}$ are secondary to an underlying ischemic etiology and suggest possible shared pathways of dysfunction. It is important that the genesis of bipolar 
disorder and correlations with CADASIL are delineated. The NOTCH3 (CADASIL) was not confirmed as a candidate gene for bipolar disorder 15 years ago, ${ }^{24}$ but a recent reanalysis indicated that there is a replicable genome-wide association of Notch signaling pathways and bipolar disorder. ${ }^{25}$ In rats, valproate activates the NOTCH3/c-FLIP signaling cascade and promotes vascular smooth muscle cell survival, attenuating development of white matter hyperintensities. ${ }^{26}$ It has also been reported that valproate controls migraines in patients with CADASIL, ${ }^{27}$ raising the possibility that valproate, the classic mood-stabilizing drug, may represent a novel drug therapy for CADASIL. ${ }^{26}$

Given the coincidental symptomatology, leukoaraiosis, and genetics of bipolar disorder and CADASIL, our report indicates that bipolar II disorder may be a poorly recognized manifestation of CADASIL. We propose that any CADASIL patient presenting with mood disturbances undergo a detailed medical history of prior manic or hypomanic episodes. Additionally, whenever a marked leukoencephalopathy is detected in bipolar disorder, CADASIL should be considered as a possible differential diagnosis, particularly when lateonset affective patients possess a family history of stroke, migraine, or dementia.

\section{Acknowledgment}

The authors thank the patient for her cooperation and understanding of this research.

\section{Disclosure}

The authors report no conflicts of interest in this work.

\section{References}

1. Chabriat H, Joutel A, Dichgans M, Tournier-Lasserve E, Bousser MG. Cadasil. Lancet Neurol. 2009;8(7):643-653.

2. Joutel A, Corpechot C, Ducros A, et al. Notch3 mutations in CADASIL, a hereditary adult-onset condition causing stroke and dementia. Nature. 1996;383(6602):707-710.

3. Dichgans M, Mayer M, Uttner I, et al. The phenotypic spectrum of CADASIL: clinical findings in 102 cases. Ann Neurol. 1998;44(5):731-739.

4. Chabriat H, Bousser MG. Neuropsychiatric manifestations in CADASIL. Dialogues Clin Neurosci. 2007;9(2):199-208.

5. Valenti R, Pescini F, Antonini S, et al. Major depression and bipolar disorders in CADASIL: a study using the DSM-IV semi-structured interview. Acta Neurol Scand. 2011;124(6):390-395.

6. Valenti R, Poggesi A, Pescini F, Inzitari D, Pantoni L. Psychiatric disturbances in CADASIL: a brief review. Acta Neurol Scand. 2008; 118(5):291-295.

7. Leyhe T, Wiendl H, Buchkremer G, Wormstall H. CADASIL: underdiagnosed in psychiatric patients? Acta Psychiatr Scand. 2005;111(5): 392-396; discussion 396-397.
8. Dericioglu N, Vural A, Agayeva N, Basar K, Anil Yagcioglu AE, Gursoy-Ozdemir Y. Cerebral autosomal dominant arteriopathy with subcortical infarcts and leukoencephalopathy (CADASIL) in two siblings with neuropsychiatric symptoms. Psychosomatics. 2013;54(6): 594-598.

9. Benazzi F. Bipolar II disorder: epidemiology, diagnosis and management. CNS Drugs. 2007;21(9):727-740.

10. Doran CM. The Hypomania Handbook: The Challenge of Elevated Mood. Philadelphia, PA: Lippincott Williams \& Wilkins; 2008.

11. Novick DM, Swartz HA, Frank E. Suicide attempts in bipolar I and bipolar II disorder: a review and meta-analysis of the evidence. Bipolar Disord. 2010;12(1):1-9.

12. Goffin KC, Dell'Osso B, Miller S, et al. Different characteristics associated with suicide attempts among bipolar I versus bipolar II disorder patients. J Psychiatr Res. 2016;76:94-100.

13. Post RM, Altshuler LL, Kupka R, et al. Illnesses in siblings of US patients with bipolar disorder relate to multigenerational family history and patients severity of illness. $J$ Affect Disorders. 2017;207: 313-319.

14. Soares J. 773-Neuroimaging abnormalities identify neuroprogressive course of bipolar disorder. Biol Psychiat. 2017;81(10):S314.

15. You J, Liao S, Zhang F, Ma Z, Li G. First report of Arg587Cys mutation of Notch3 gene in two Chinese families with CADASIL. J Stroke Cerebrovasc Dis. 2017;26(1):e1-e4.

16. Judd LL, Akiskal HS, Schettler PJ, et al. A prospective investigation of the natural history of the long-term weekly symptomatic status of bipolar II disorder. Arch Gen Psychiatry. 2003;60(3):261-269.

17. Nasrallah HA. Consequences of misdiagnosis: inaccurate treatment and poor patient outcomes in bipolar disorder. J Clin Psychiatry. 2015; 76(10):e1328.

18. Marlinge E, Bellivier F, Houenou J. White matter alterations in bipolar disorder: potential for drug discovery and development. Bipolar Disord. 2014;16(2):97-112.

19. Saricicek A, Zorlu N, Yalin N, et al. Abnormal white matter integrity as a structural endophenotype for bipolar disorder. Psychol Med. 2016; 46(7):1547-1558.

20. Redlich R, Almeida JJ, Grotegerd D, et al. Brain morphometric biomarkers distinguishing unipolar and bipolar depression. A voxelbased morphometry-pattern classification approach. JAMA Psychiatry. 2014;71(11):1222-1230.

21. Mwangi B, Wu MJ, Cao B, et al. Individualized prediction and clinical staging of bipolar disorders using neuroanatomical biomarkers. Biol Psychiatry Cogn Neurosci Neuroimaging. 2016;1(2):186-194.

22. Spuhler K, DeLorenzo C, Parsey R, Huang C. 933-Entropy analysis shows temporal pole diffusivity changes in bipolar disorder. Biol Psychiat. 2017;81(10):S377-S378.

23. Di Donato I, Bianchi S, De Stefano N, et al. Cerebral autosomal dominant arteriopathy with subcortical infarcts and leukoencephalopathy (CADASIL) as a model of small vessel disease: update on clinical, diagnostic, and management aspects. BMC Med. 2017;15(1):41.

24. Ahearn EP, Speer MC, Chen YT, et al. Investigation of Notch3 as a candidate gene for bipolar disorder using brain hyperintensities as an endophenotype. Am J Med Genet. 2002;114(6):652-658.

25. Pedroso I, Lourdusamy A, Rietschel M, et al. Common genetic variants and gene-expression changes associated with bipolar disorder are over-represented in brain signaling pathway genes. Biol Psychiatry. 2012;72(4):311-317.

26. Yuan P, Salvadore G, Li X, et al. Valproate activates the Notch3/c-FLIP signaling cascade: a strategy to attenuate white matter hyperintensities in bipolar disorder in late life? Bipolar Disord. 2009;11(3):256-269.

27. Martikainen MH, Roine S. Rapid improvement of a complex migrainous episode with sodium valproate in a patient with CADASIL. J Headache Pain. 2012;13(1):95-97. 
Neuropsychiatric Disease and Treatment

Dovepress

\section{Publish your work in this journal}

Neuropsychiatric Disease and Treatment is an international, peerreviewed journal of clinical therapeutics and pharmacology focusing on concise rapid reporting of clinical or pre-clinical studies on a range of neuropsychiatric and neurological disorders. This journal is indexed on PubMed Central, the 'PsycINFO' database and CAS,

and is the official journal of The International Neuropsychiatric Association (INA). The manuscript management system is completely online and includes a very quick and fair peer-review system, which is all easy to use. Visit http://www.dovepress.com/testimonials.php to read real quotes from published authors.

Submit your manuscript here: http://www.dovepress.com/neuropsychiatric-disease-and-treatment-journal 